\title{
Distributed Traffic Balancing Routing for LEO Satellite Networks
}

\author{
Yong Lu, Fuchun Sun, Youjian Zhao, Hongbo Li, Heyu Liu \\ Tsinghua university, Beijin, China \\ \{lysky007@gmail.com, fcsun@tsinghua.edu.cn, zhaoyoujian@tsinghua.edu.cn \\ ,hbli@mail.tsinghua.edu.cn ,hy-liu10@mails.tsinghua.edu.cn \}
}

\begin{abstract}
Satellite networks have been widely investigated both in the business and academia for many years, with many important routing algorithms reported in the literatures. However, fewer existing routing algorithms focus on the trade-off between the routing survivability and the routing computation and storage overheads. Due to topological dynamics, it is difficult to effectively apply the conventional routing protocols such as RIP or OSPF to Low Earth Orbit (LEO) satellite networks. According to the virtual topology model based on virtual node, this paper propose a new fully distributed routing protocol for LEO satellite networks, called Distributed Traffic Balancing Routing (DTBR). The proposed protocol not only guarantees the routing survivability and provides the ability of traffic balancing, but also result in few additional computation and storage overheads only deriving from the information flooding of failed satellites. Simulation results demonstrate positive conclusions of our methods.
\end{abstract}

Index Terms - Low Earth Orbit (LEO), satellite networks, snapshot, survivability

\section{INTRODUCTION}

Satellite networks have been an important infrastructure of both Next-Generation Internet (NGI) and Interplanetary Internet (IPN). Compared with the ground networks, satellite networks can provide the extraordinary advantages in global communication, broadcast, space information development, etc. Most satellite networks make use of inter-satellite links (ISLs) to construct a dynamic network, whose characteristics such as the topological dynamics, limited processing facility and storage space, etc., obstruct the effective implementation of conventional routing protocols such as RIP or OSPF. With the rapid development of space technologies, future space communication will be confronted with complex situation. Not only the natural factors such as electromagnetic interference, physical failure and energy limited, but also the factitious behavior such as spatial competition, military strike, etc., might result in the satellite failure, which can bring the significant impact on the communication of satellite networks. First of all, the effects of the failed satellites on communication are global. Once the failed satellites move to a new geographical area where previous satellites are safe, the communication of this area is influenced until the failed satellites move away. Secondly, due to the long renewing cycle and expensive maintenance, the recovery of the failed satellites needs a long time or can not even be completed. Therefore it is a challenge issue to develop the efficient and survivable routing protocols for satellite networks.

So far, many routing schemes have been developed for satellite networks. For the routing algorithms based on virtual topology (VP) [1], [2], [3], the time varying topology is tackled by a discrete time network model. In each time interval, the satellite network is assumed to have a fixed topology. The time interval is determined by the change of the physical topology or other metrics such as propagation delay [1]. For example, the concept of snapshots [2] was introduced to describe the dynamics of LSNs. When a new ISL is added or an already existing ISL is broken, a new snapshot different from the previous one will be formed. However, the number of snapshots in its cycle can be very large as the ISLs change pretty fast when they pass the south and north Polar Regions or switch over the seam, which results in the very large overheads of routing computation and storage. In [4], based on the predictable changes of spacecraft networks, the authors formalize the snapshot concept. Every satellite only stores the differences between adjacent snapshots and the transition rules, so the snapshot transition can be completed automatically by the satellites with low storage overhead. Nonetheless, the snapshot number is essentially unchanged, which means that the frequent routing computation is still unavoidable. With the rapid growth of Internet-based applications, many distributed routing algorithms [5], [6], [7] are presented to forward the packets with the nominal overhead. Since the distributed routing schemes decides the route by the local information, they are just suitable for the case that only a neighboring satellite fails, instead of the case that any satellite may fail at any time. To improve the routing performance, routing protocols based on multi-layered satellite network are proposed [8], [9], [10]. However, these routing protocols only considered the case of random node failures in LEO satellite layer. In fact, if the MEO satellite fails, these routing protocols will collapse. An agent-based load balancing routing (ALBR) [11], [12] is presented for LEO satellite networks, in which mobile agents and stationary agents cooperate to achieve the load balancing routing. However, the case of random node 
failure is not considered. In this case, the mobile agent does not know the network topology, so it is possible that the mobile agent remove itself before it reaches the destination because of the faulty satellites. From the above statement, it can be seen that the existing routing schemes for satellite networks can't reach a trade-off between the survivable ability and the routing overheads. The routing protocols based on VP can update routing tables for every snapshot after getting the information of faulty satellites; however, it suffers from the slow reaction and the large computation and storage overheads. Although the distributed routing protocols introduce low computation and storage overhead, the routing survivability is limited. Therefore, it is necessary to develop a routing scheme with low computation and storage overheads and good routing survivability for satellite networks.

In this paper, based on the virtual topology model [13], we propose a new routing protocol for Low Earth Orbit (LEO) satellite networks, called Distributed Traffic Balancing Routing (DTBR). The proposed protocol not only guarantees the routing survivability and provides the ability of traffic balancing, but also result in few additional communication overheads only deriving from the information flooding of failed satellites. The performance of DTBR is also evaluated by simulation.

The rest of this paper is organized as follows: Section 2 presents the system model of LEO satellite networks and Section 3 introduces the virtual topology model for LEO satellite networks. The Distributed Traffic Balancing Routing (DTBR) protocol is proposed in Section 4 and Section 5 presents simulation results. We summarize the paper in Section 6.

\section{SYSTEM MODEL}

The LEO Satellite Network (LSN) is the time varying and predictable network composed of $N$ orbits, each orbit with $M$ satellites. There are two constellation designs for satellite networks: Walker delta and Walker star constellations, which can be denoted by $N \times M / M / F$, where $F=0, \ldots, N-1$, as shown in Figure 1. For Walker delta constellation, the orbit inclination angle is less than $90^{\circ}$, and the planes are separated from each other with the same angular distance of $360^{\circ} / N$. For Walker star constellation, the orbit inclination angle is near $90^{\circ}$, and the planes are separated from each other with the same angular distance of $180^{\circ} / N$. The Inter-plane ISLs connect satellites from different orbits, while the Intra-plane ISLs connect satellites in the same orbit.

In this work, we focus on the LSN with the Walker star constellation, i.e., Polar Orbit satellite networks, where the Intra-plane ISLs are maintained at all times, while the Inter-plane ISLs are shut down in the Polar Regions and re-established outside of the Polar Regions. The satellites between the adjacent $0^{\text {th }}$ and $(\mathrm{N}-1)^{\text {th }}$ planes move in opposite directions, which results in a called seam existing between the two planes. It is expensive to connect two satellites over the seam [14], so we assume that there are not cross-seam ISLs. The LSN can be formalized as a time graph $G(t)=(V(t), E(t))$, where $V(t)$ and $E(t)$ are respectively the set of nodes and edges at time $t$. In the random case, any satellite might fail at any time, so $|V(t)|$ is not always equal to $N \times M$. The concept of snapshot [2] can capture the dynamics of $G(t)$. In this concept, once a link is re-established or a link is closed, a snapshot different from the previous one is formed. For the periodicity of satellite movement, the LSN topology can be described by a sequence of snapshots. The number of snapshots in an orbit cycle is very large because of the frequent changes of the Interplane ISLs in the Polar Regions.

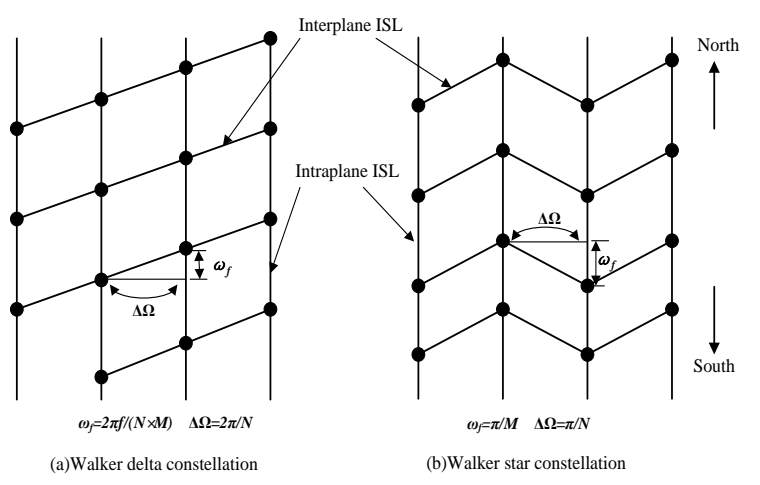

Figure1 Satellite networks constellation

\section{VIRTUAL TOPOLOGY}

\section{A. Virtual topology and address mapping}

Based on the Earth-fixed footprint mode of the satellite antenna systems on Polar orbit LSN, the authors formalized the LSN as a virtual topology FVT [13], Compared with the physical topology of LSN, the FVT exhibits some important advantages such as few snapshots, well-proportioned snapshot length, avoidance of path stretch and contraction, which is better for the realization of routing protocols. Let $G_{v}(t)$ denote the topological graph of FVT, firstly, two basic mappings are necessary for performing the routing protocol on FVT: the mapping from the LSN to FVT can construct $G_{v}(t)$, and the mapping from the earth to FVT can determine the logical location of the earth terminals. We use a pair of ordered integers $(x(c), y(c))$ to identify the actual location of satellite $\mathrm{c}$ on the LSN, where $\mathrm{x}(\mathrm{c})$, for $\mathrm{x}(\mathrm{c})=0, \ldots, \mathrm{N}-1$ is the orbit number and $y(c)$, for $y(c)=0, \ldots, M-1$ is the satellite number. Let $\alpha(\mathrm{s}, \mathrm{t})$ and $\beta(\mathrm{s}, \mathrm{t})$ denote the logical location of node $\mathrm{s}$ at time $t$, where $t$ is the time that the satellite has moved. The logical location of any satellite $(\mathrm{x}(\mathrm{c}), \mathrm{y}(\mathrm{c}))$ at time $t$ is described as follows:

$$
\begin{aligned}
& \alpha(c, t)=x(c) \\
& \beta(c, t)=[y(c)+\lfloor[w(t \% T)] /(2 \pi / M)\rfloor] \% M
\end{aligned}
$$

Where $\omega, T$ and $M$ are the angle velocity of the satellites, the orbital cycle, and the satellite number of the plane, respectively. 
Since $G_{v}(t)$ is independent of the rotation of the earth, we assume the geographic coordinate system is constructed on $G_{v}(t)$ at $t=0$. The mapping from the earth to $G_{v}(t)$ can be achieved by computing new geographic location on $G_{v}(t)$ at any time $t$. Let $\operatorname{lon}(E, t)$ and $\operatorname{lat}(E, t)$ respectively denote the longitude and latitude of the earth node $\mathrm{E}$ at time $t$ on $G_{v}(t)$, which are computed as follows:

$$
\begin{aligned}
& \operatorname{lon}(E, t)= \begin{cases}\operatorname{lon}_{t}=\left[\operatorname{lon}(E, 0)+\left(t \% T^{\prime}\right) \omega^{\prime}\right] \%(2 \pi) \\
2 \pi-\operatorname{lon}_{t} & \operatorname{lon}_{t}>\pi \\
\operatorname{lon}_{t} & \operatorname{lon}_{t} \leq \pi\end{cases} \\
& \operatorname{lat}(E, t)=\operatorname{lat}(E, 0)
\end{aligned}
$$

Where $\omega^{\prime}$ and $\mathrm{T}^{\prime}$ are the angle velocity and the cycle of the rotation of the earth, respectively. According to Formula (2), the logical location of any node $E$ on the earth at time $t$ is computed below.

$$
\begin{aligned}
& \alpha(E, t)=X_{0}(\operatorname{lon}(E, t)) \\
& \beta(E, t)=Y_{0}(\operatorname{lon}(E, t), \operatorname{lat}(E, t))
\end{aligned}
$$

Where $X_{0}$ (lon) and $Y_{0}$ (lon,lat) is the mapping from any location on the earth to $G_{v}(t)$ at $t=0$, which can be computed beforehand.

\section{B. Virtual snapshot transition}

According to the properties of FVT [13], a prominent superiority of the FVT is the maximal number of the snapshots is not more than the footprint number of a plane no matter how many faulty satellites happen. Besides, for the strict orbital movements of all satellites, the transition between virtual snapshots has also the specific regularity. Since the FVT can result in few snapshots and its topological dynamics can be predetermined due to the strict orbital movement of all satellites, it is possible to realize a fully distributed routing protocol. In fact, every satellite can independently calculate current $G_{v}(t)$ at any time $t$ as long as it owns the information of all failed satellites, which includes two parts: the information flooding of failed satellites and execution of a snapshot transition algorithm. The first part enables every safe satellite to get the location of all failed satellites, described as follows: every satellite reports all failed satellites it knows to its safe neighbors, and the same information is only sent once; for the fast updating, the flooding message is run by the highest priority queues. When a safe satellite gets the logical location of all failed satellites by Formula (1), the snapshot transition is executed by the following Algorithm.

\section{Snapshot Transition $\operatorname{ST}\left(c, t_{0}, t\right)$}

Takes failed satellite $c$, the time $t_{0}$ of the last snapshot transition and current time $t$ as input

1: if running snapshot transition first time

2: $\quad \mathrm{t}_{0} \leftarrow \mathrm{t}$

3: end if

$4:$ if $\beta\left(\mathrm{c}, \mathrm{t}_{0}\right) !=\beta(\mathrm{c}, \mathrm{t})$
5: Set all intraplane $V L s$ from $\left(\alpha\left(c, t_{0}\right), \beta\left(c, t_{0}\right)\right)$ to its neighbors to true;

6: if $\left(\alpha\left(c, t_{0}\right), \beta\left(c, t_{0}\right)\right)$ and its neighbors of different planes are not affected by latitude threshold

7: $\quad$ set their VLs to true;

8: else

9: $\quad$ set their VLs to false;

10: end if

11: set all $V L s$ from $(\alpha(\mathrm{c}, \mathrm{t}), \beta(\mathrm{c}, \mathrm{t}))$ to its all neighbors to false;

12: $\mathrm{t}_{0} \leftarrow \mathrm{t}$;

13: else

14: set all $V L s$ from $(\alpha(\mathrm{c}, \mathrm{t}), \beta(\mathrm{c}, \mathrm{t}))$ to its all neighbors to false;

15: end if

16: end if

The snapshot transition is executed once the satellite switches to next logical region. Algorithm 1 firstly judges whether the snapshot transition was run first time. If it holds, the transition will be executed by Step 14; otherwise the transition includes two processes: the $V L$ restoration of time $t_{0}$ and the $V L$ destruction of current time $t$, which are executed by Step 5 10 and Step 11, respectively. A safe LEO satellite needs to run Algorithm $1 N_{F}$ times when it enters a new logical region, where $N_{F}$ is the number of all failed LEO satellites.

\section{ROUTING PROTOCOL}

In this section, we will present a new routing protocol with traffic prediction, called Distributed Traffic Balancing Routing (DTBR), which not only provides good routing survivability with low load, but introduces an ISL cost factor to avoid congestion and balance the traffic. Besides, the protocol will not result in any additional communication overheads except the flooding of failed satellites.

\section{A. ISL cost}

For the routing quality, a cost factor $\lambda$ based on the empirical dispersion of global hot spot zones is introduced to the ISL cost, which dynamically adjusts the traffic distribution for traffic balancing. The ISL cost metric is presented as:

$I S L_{\cos t}=\lambda P D(V L)$

Where $P D(V L)$ present the propagation delay of virtual link $V L$. Let $\delta(F)$ denotes the center of logical region $F$. $P D(V L)$ is defined as follows.

Definition 1 Let $V l\left(\mathrm{~F}_{\mathrm{i}, \mathrm{j}}, \mathrm{F}_{\mathrm{k}, \mathrm{r}}\right)$ be the virtual link between logical region $F_{i, j}$ and $F_{k, r}$ in $G_{v}(t)$. The delay function $\operatorname{PD}\left(\operatorname{Vl}\left(\mathrm{F}_{\mathrm{i}, \mathrm{j}}, \mathrm{F}_{\mathrm{k}, \mathrm{r}}\right)\right)$ is defined as: 


$$
P D\left(V l\left(F_{i, j}, F_{k, r}\right)\right)=\left\{\begin{array}{c}
\text { propagation delay from } \delta\left(F_{i, j}\right) \text { to } \delta\left(F_{k, r}\right), \\
\exists I S L\left(S\left(F_{i, j}\right), S\left(F_{k, r}\right)\right) \wedge \\
(0 \leq i, k<N) \wedge(0 \leq j, r<M) \\
\infty \quad \text { otherwise }
\end{array}\right.
$$

Due to population dispersion, economy and technology development, most of hot spots locate at the Northern Hemisphere, especially within the scope of $50^{\circ} \mathrm{N}$ [15]. The forecasted traffic over LEO satellite systems in 2005 is illustrated in Figure 2. The ISL cost modification factor in [11] only considers the north hemisphere. Since the south hemisphere still has some hop spots, we modify the ISL cost modification factor as follows:

$$
\lambda= \begin{cases}e^{\text {lat } / 90} & 0<\text { lat }<50 \\ e^{\mid \text {lat } \mid / 90} & (\text { lat }<0) \wedge\left(\left(-80^{\circ}<\text { lon }<-40^{\circ}\right)\right. \\ & \vee\left(-15^{\circ}<\text { lon }<50^{\circ}\right) \vee \\ & \left.\left(110^{\circ}<\text { lon }<150^{\circ}\right)\right) \\ e^{-\mid \text {lat } \mid / 90} & \text { otherwise }\end{cases}
$$

Where lat and lon respectively represent the latitude and longitude of the LEO satellite. Based on $\lambda$ factor, the ISL cost in hot spot zones tends to increase, and in nonhot spot zones tends to decrease.

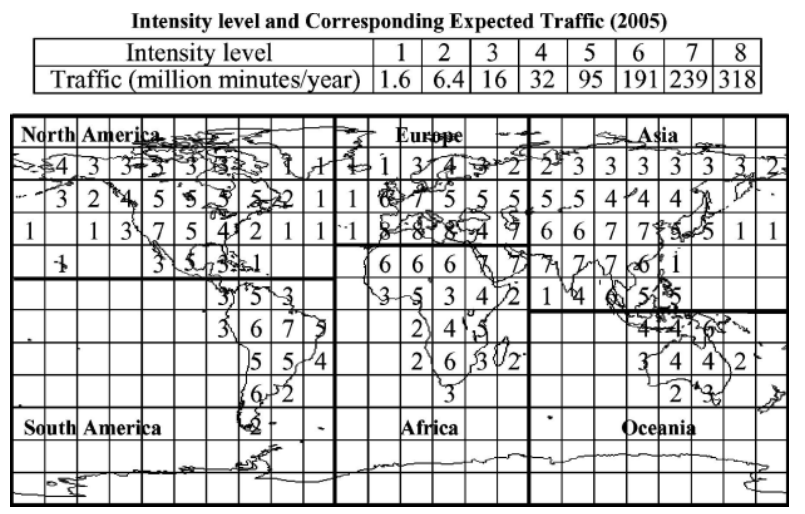

Figure2.Earth zone division and user density levels

\section{B. Routing calculation}

Since any LEO satellite can automatically get current FVT and the ISL costs, the routing table can be calculation independently by every LEO satellite anytime. For the earth's rotation, the hot spots will continuously switch to different logical regions. To timely reflect the traffic change on FVT, once the LEO satellite switches to next logical region, its routing table should be updated, which is calculated as follows:

Step 1: Once the satellite switches to next logical region, if failed satellites exist, every LEO satellite automatically finishes snapshot transition by Algorithm 1; otherwise its snapshot keeps constant in terms of the property of $F V T$ [13].

Step 2: Every LEO satellite computes $I S L_{\text {cost }}$ of all ISLs by formula (4). In fact, $P D(V L)$ of all virtual links is only computed once if there is not the failed satellites; otherwise only the virtual links affected by the failed satellites are set to $\infty$. For balancing factor $\lambda$, every satellite needs to compute the geographical coordination of all satellites on the earth, so the time complexity is $\mathrm{O}(n)$, where $n$ is the number of all safe satellites.

Step 3: Once the satellite gets the ISL costs of all ISLs, the routing table is updated as follows:

\section{Routing updating}

1: var $\mathrm{U}(\mathrm{F}, \mathrm{S}) /^{*}$ The updating mark of virtual snapshot $\mathrm{S}$ in footprint $\mathrm{F} *$ /

2: $\operatorname{var} \mathrm{R}(\mathrm{F}, \mathrm{S}) /^{*}$ The routing computation mark of footprint $\mathrm{F}$ for virtual snapshot $\mathrm{S} * /$

3: if $(\mathrm{U}(\mathrm{F}, \mathrm{S}))$ // The virtual snapshot $\mathrm{S}$ in footprint $\mathrm{F}$ is updated in current footprint

4: $\quad$ if $(\mathrm{R}(\mathrm{F}, \mathrm{S}))$ // The routing table for new virtual snapshot $\mathrm{S}$ in footprint $\mathrm{F}$ has been updated

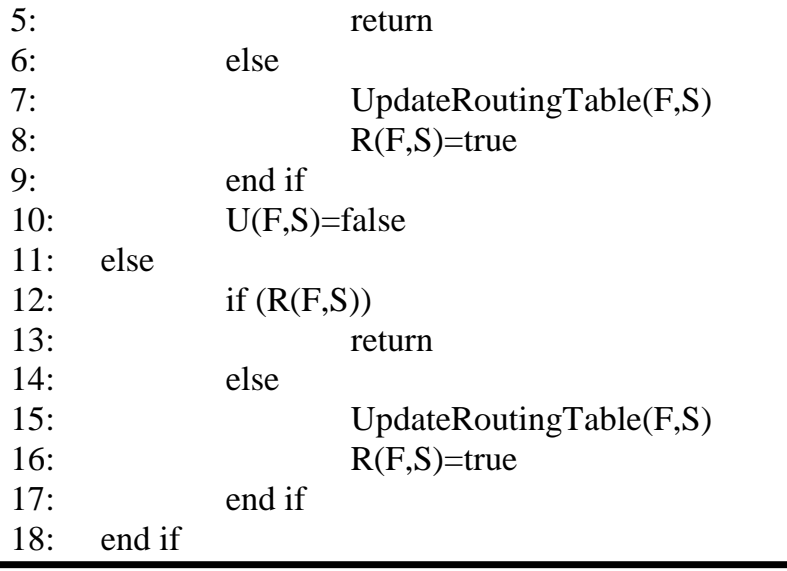

The routing table is calculated by Dijkstra algorithm with time complexity $\mathrm{O}\left(n^{2}\right)$, where $n$ is the number of all safe satellites.

\section{Congestion avoidance}

The DTBR uses the traffic prediction factor $\lambda$ to avoid congestion, whereby the traffic deviates from the hot spot zones to non-hot spot zones. In this way, the congestion probability is decreased. However, the congestion still may occur when the burst traffic is generated. In this case, the ELB scheme [16] is used, whereby a congested satellite sends a signal to its neighboring satellites to decrease their sending rates, and its neighbors search for alternate paths.

\section{Satellite failure}

For satellite failures, the end-to-end path in LSN does not always exist. For example, once failed satellites move to source or destination location, data transmission between them will fail. In this case, two methods can be considered: store-carry-forward mechanism of DTN [17] and source waiting. In the first method, when the end-toend path does not exist, the packets can be routed to an intermediate node where the packet will wait until there is a feasible path. Due to the predictability about future topology, the packets can be forwarded successfully as long as the end-to-end path exists in the future. The shortage of this method lies in that it presents high 
requirement on the satellite's storage space. For the second method, before the earth terminal sends the packets, it enquires of the current satellite whether the destination is reachable. If yes, the packets will be forwarded; otherwise the terminal will wait until a satellite can serve it and a feasible path exists. Since the waiting delay is far greater than the transmission delay, and most existing LEO satellites are limited to the storage space, the second method is a suitable selection.

\section{PERFORMANCE EVALUATION}

To demonstrate the effectiveness of our routing schemes, three main experiments are constructed based on the ns2 simulator. Four routing protocols are involved in the experiments as comparison, which include DTBR without source waiting, DTBR with source waiting, Datagram Routing Algorithm (DRA) [5] and Satellite Snapshot Routing (SSR). In SSR, the routing table with minimal propagation delay is calculated by Dijkstra algorithm for every snapshot. In the simulation scenario, a polar orbit LEO satellite constellation with 12 planes and 24 satellites in each plane is generated. The ISL capacity is $2.5 \mathrm{Mb}$ and latitude threshold is $75^{\circ}$. In all experiments, the source-destination pair is located at $\left(37.9^{\circ} \mathrm{N}, 31.6^{\circ} \mathrm{E}\right)$ and $\left(30^{\circ} \mathrm{S}, 100^{\circ} \mathrm{W}\right)$, and simulation time is $6794 \mathrm{~s}$, i.e., an orbit cycle.

To evaluate the routing survivability, we generate randomly $0 \sim 30 \%$ failed satellites in the system by the interval $5 \%$ and set a low data rate with one packet per 30s. Figure 3 illustrates the loss packet rate. Since DRA forwards the packets in terms of local information, its loss packet rate dramatically increases as the fault rate is raised. On the contrary, for DTBR and SSR, the packet is discarded if and only if the end-to-end path does not exist. DTBR with source waiting can successfully route all packets even if the fault rate reaches $30 \%$. Theoretically, as long as there is a safe satellite, the packets can be forward successfully with the arbitrary delay since the satellite might move to any location on the earth. As shown in Figure 4, both DTBR without source waiting and SSR have the similar average end-to-end delay. For the predominant waiting delay, it is inevitable that DTBR with source waiting suffers from large end-to-end delay as fault rate is raised. Figure 5 illustrates the changes of average waiting delay in different fault rate.

To evaluate the throughput, all satellites over hot spot zones generates on/off traffic whose Pareto distribution with the average on/off durations equal to $100 \mathrm{~ms}$. The traffic bit rate of source node is varied from 200 to 1000 by $200 \mathrm{~kb} / \mathrm{s}$. Figure 6 presents the average packet loss rate of DTBR and SSR. Due to ISL cost factor $\lambda$, the traffic can be detoured from the hot spots by DTBR, which decreases the probability of packet loss. Figure 7 shows the throughput of DTBR and SSR. It is obvious that DTBR has a better throughput than SSR for less packet loss rate when the terminal bit rate is increased.

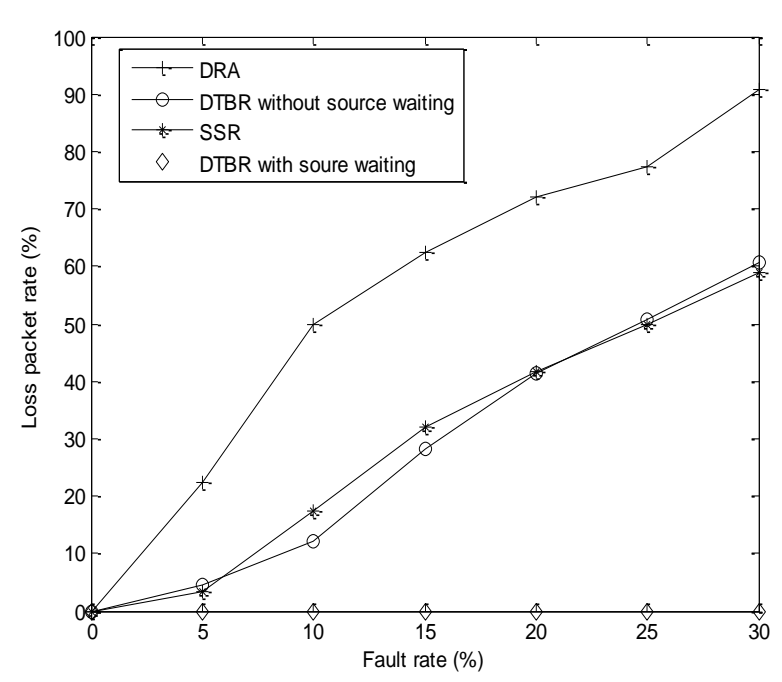

Figure 3.The loss packet rate
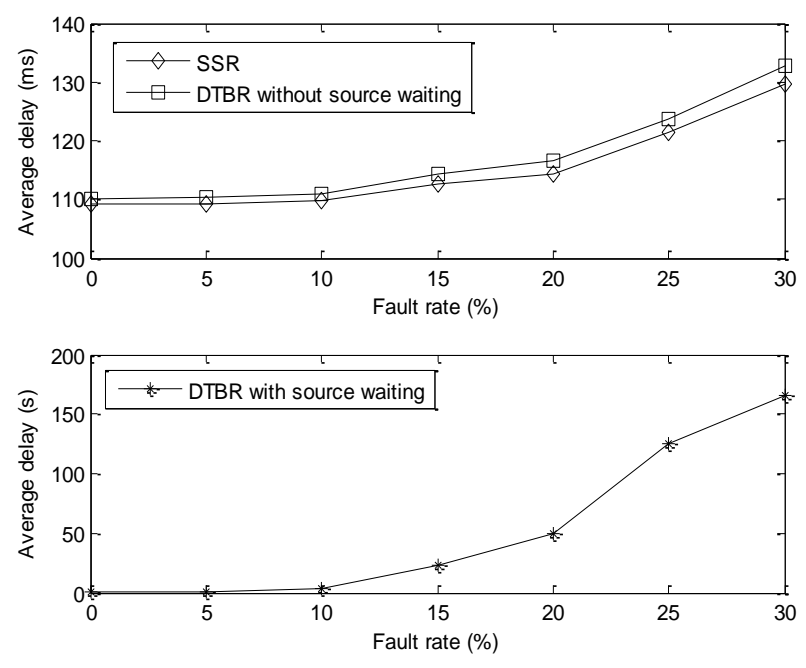

Figure4.The average end-to-end delay

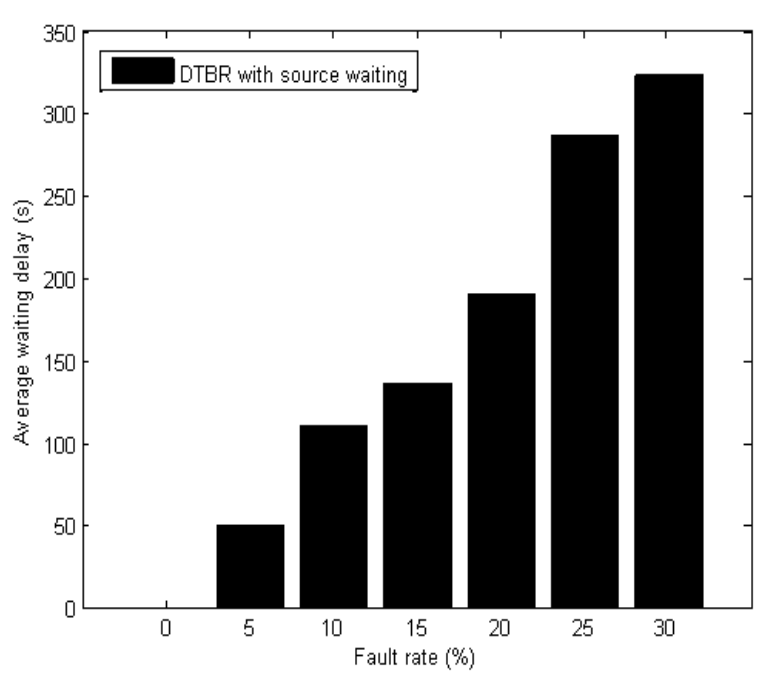

Figure5.The average waiting delay of DTBR 


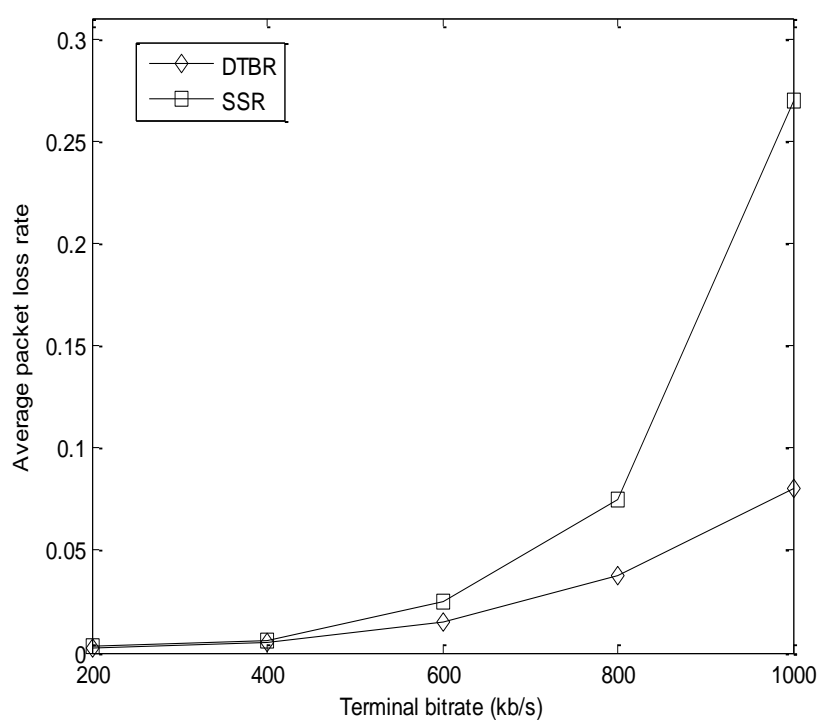

Figure6.The average packet loss rate

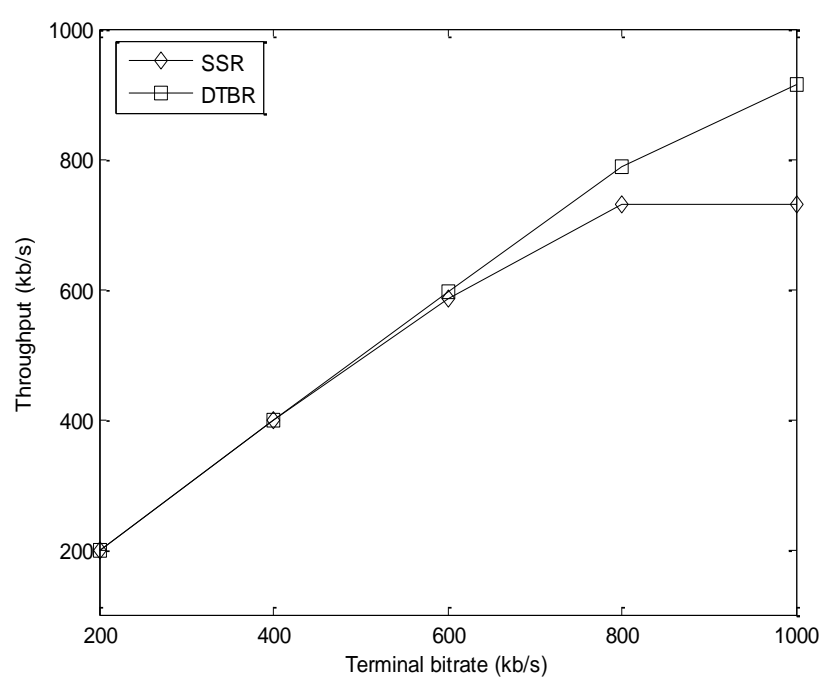

Figure 7.The throughput results

\section{CONCLUSIONS}

Based on the virtual topology model [13], this paper proposes a distributed traffic balancing routing protocol for LEO satellite networks, which provides the routing survivability, the ability of traffic balancing, and few additional communication overheads only deriving from the information flooding of failed satellites. Simulation results demonstrate positive conclusions of the proposed protocol.

\section{ACKNOWLEDGMENT}

This work was supported by the National Basic Research Program of China (973 Program) (Grant No: 2012CB821206), National Natural Science Foundation of China (Grant No: 60903184, 61073167), the National High Technology Development Program of China (Grant No: 2011AA010704), Beijing Natural Science
Foundation (Grant No: 4122037), National science and technology support program of China (Grant No: 2011BAH15B08).

\section{REFERENCES}

[1] Werner M, A dynamic routing concept for ATMbased satellite personal communication networks, IEEE Journal on Selected Areas in Communications, 1997, 15(8):1636-1648.

[2] Gounder, V.V., Prakash, R., Abu-Amara, H, Routing in LEO-based satellite networks, In: Richardson, TX. Proc of the Wireless Communications and Systems Workshop, 1999, 2211-2216.

[3] Chang, H. S, Kim, B. W, Lee, C, FSA-based link assignment and routing in low-earth orbit satellite networks, IEEE Transaction on Vehicular Technology, 1998, 47(3):1037-1048.

[4] Fischer D,Basin D,Engel T, Topology dynamics and routing for predictable mobile networks. In:Proc.of the ICNP 2008. Orlando: IEEE Communications Society 2008:207-217.

[5] Henderson, T., \& Katz, H, On distributed geographic based packet routing for LEO satellite networks, In San Francisco, USA. Proc of IEEE globecom, 2000, 1119-1123.

[6] E. Ekici, I. F. Akyildiz, and M. D. Bender, A Distributed Routing Algorithm for Datagram Traffic in LEO Satellite Networks, IEEE/ACM Trans. Networking, 2001, 9(2):137-147.

[7] Sanctis, M. D., Cianca, E., \& Ruggieri, M, IP based routing algorithm for LEO satellite networks in near polar orbits, In: Montana, USA. Proc of the IEEE aerospace conference, 2003, 1273-1280.

[8] Chen, C. and Ekici, E., A Routing Protocol for Hierarchical LEO/MEO Satellite IP Networks, ACM/Kluwer Wireless Networks Journal (WINET) 2005;11(4):507-521.8

[9] Fei Long, Naixue Xiong, Athanasios V. Vasilakos, Laurence T. Yang, Fuchun Sun, A sustainable heuristic QoS routing algorithm for pervasive multilayered satellite wireless networks, J. Wireless Networks 2010;16(6):1657-1673.

[10] Yunhui Zhou, Fuchun Sun, Bo Zhang, A novel QoS routing protocol for LEO and MEO satellite networks, Int. J. Satell. Commun. Network. 2007; 25:603-617.

[11] Yuan Rao, Ru-chuan Wang, Agent-based load balancing routing for LEO satellite networks, computer networks, 2010, 54(17):3187-3195.

[12] Gao Zihe, Guo Qing, Na Zhenyu, Distributed Routing Algorithm with Traffic Prediction in LEO Satellite Networks, J. Information Technology, 2011, 10(2):285-292.

[13] Yong. Lu, Fuchun Sun, Youjian Zhao, Virtual topology for LEO satellite networks based on Earthfixed footprint mode, IEEE Communications Letters, 2013, 17(2):357-360.

[14]Ferreira, J., \& Galtier, J, Topological design, routing and hand-over in satellite networks, Handbook of 
Wireless Networks and Mobile Computing London: Wiley, 2005, 473-507.

[15] A. Svigelj, M. Mohorcic, G. Kandus, Routing in ISL networks considering empirical IP traffic, IEEE Journal on Selected Areas in Communications 2004, 22(2):261-272.

[16] T. Tarik, M. Daisuke, A. Jamalipour, Explicit load balancing technique for NGEO satellite IP networks with on-board processing capabilities, IEEE/ACM Transactions on Networking, 2009, 17(1): 281-293.

[17]Fall K. A delay-tolerant network architecture for challenged Internets, In: Proc. of the ACM SIGCOMM. Karlsruhe: ACM Press, 2003, 27-34.

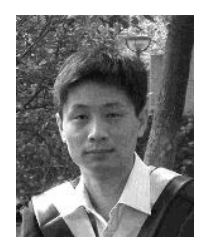

Yong Lu, received the B.S. degree from Air force college of Guilin, China, in 1998, the MS degree in University of Electronic Science and Technology of china, in 2007. $\mathrm{He}$ is currently a $\mathrm{PhD}$ candidate of Tsinghua University in china. His research interests include fault-tolerant computing, routing protocols and satellite networks.

Fuchun Sun, Professor in the Department of Computer Science and Technology, Tsinghua University. His research interests include neural-fuzzy systems, variable structure control, networked control systems, and robotics.

Youjian Zhao, Professor in the Department of Computer Science and Technology, Tsinghua University. His current interests include computer network architecture, high speed routers, and large capacityswitch fabric.

Hongbo Li, Assistant professor with the Department of Computer Science and Technology, Tsinghua University. His research interests include networked control systems and intelligent control.

Heyu Liu, Ph. D. candidate in Tsinghua university from China. His research interests include routing protocols and satellite networks.

How to cite this paper: Yong Lu, Fuchun Sun, Youjian Zhao, Hongbo Li, Heyu Liu,"Distributed Traffic Balancing Routing for LEO Satellite Networks", IJCNIS, vol.6, no.1, pp.19-25,2014. DOI: 10.5815/ijcnis.2014.01.03 